\title{
Train Control Algorithm Based on Generalized Predictive Control
}

\author{
Jiaxi Yuan ${ }^{1}$, Xiangxian Chen ${ }^{1}$, Chenge Geng ${ }^{1}$, Zhujun Ling ${ }^{2}$, Dandan $\mathrm{Liu}^{2}$ and Guantao $\mathrm{Hu}^{2}$ \\ ${ }^{1}$ Department of Instrumentation Science and Engineering, Zhejiang University, Hangzhou, China \\ ${ }^{2}$ Research and Development Center, Zhejiang United Science and Technology Co., Ltd, Hangzhou, China
}

\begin{abstract}
The train running process is a complicated process, and the traditional control algorithm is difficult to adapt to the changing running conditions. GPC has the characteristics of multi-step prediction, rolling optimization and feedback correction, and it is a kind of control algorithm which is easy to be realized. In this paper, based on the original GPC, stair-like control scheme is used and the predicted future changes are considered as feedback. In this way, the matrix inversion operation is avoided and the overshoot is suppressed. The effectiveness of the improved GPC algorithm is proved by the simulation.
\end{abstract}

Keywords: ATO, GPC, stair-like control, suppress overshoot

\section{Introduction}

As one of the most efficient passenger transport means, urban rail transit has been widely used in different cities. In order to realize the safe, reliable and efficient operation of the train, the ATO (Automatic Train Operation) has been applied in the control of urban rail trains [1]. The most important purpose of ATO system is to adjust traction/braking force in different operation environment. Now, ATO control algorithm can be divided into classical control method, adaptive control algorithm and intelligent control algorithm, etc. PID control algorithm is the representative of the classical control algorithm, which is stable and easy to realize. However, with the running condition changing, the train traction/braking force may need to be adjusted continually [2]. The control method based on fuzzy control can improve the performance of the ATO system, but it is not easy to adjust the performance of the system online [3].

GPC (Generalized Predictive Control) is a kind of predictive control algorithm which has received extensive attention in recent years. It uses the control strategy of multi-step prediction, rolling optimization and feedback correction [4]. In this paper, based on the original GPC, stair-like control scheme and the predicted future changes are considered as feedback. The traction/braking force of the train is calculated in real time to ensure the accuracy and comfort of the running process.

\section{Train Model}

The slope of the train track is generally small, and the radius of the curve is far greater than the length of the train. In order to simplify and achieve the goal of on-line control, the train can be regarded as a single particle in this paper. The resultant force of the train is composed of traction/braking force and resistance. According to Newton's law of mechanics, the train model can be established as:

$$
\left\{\begin{array}{l}
d v / d t=c F \\
F=u-w \\
w=\alpha_{0}+\alpha_{1} v+\alpha_{2} v^{2}
\end{array}\right.
$$

\footnotetext{
${ }^{1}$ Corresponding author. Tel.: + 8615957187314 .

E-mail address: sherryueda@126.com.
} 
where, $t$ is time, $v$ is the train speed, $c$ is the acceleration coefficient, $F$ is the resultant force of train, $u$ is the traction/braking force, $w$ is the resistance; $\alpha_{0}, \alpha_{1}, \alpha_{2}$ are the resistance coefficient.

The train model is difficult to describe with a fixed linear model, the following difference model can be used to describe the model of the train.

$$
\left(1+a_{1}(k) \mathrm{z}^{-1}\right) y(k)=b_{0}(k) u(k-1)+d_{0}(k)+\delta(k)
$$

where, $y(k)$ is the train speed, $u(k)$ is the input traction/braking force, $d_{0}$ is the speed deviation parameter, $\delta(k)$ is the random noise, $a_{1}(k), b_{0}(k)$ are the model parameters.

\section{GPC Algorithm and Its Improvement}

GPC uses the CRIMA (Controlled Autoregressive Integrated Moving Average) model to describe the controlled object. Through the prediction of the future changes of the system, online computing, rolling optimization and feedback correction, so that the system can achieve the minimum error of the following [5]. GPC has excellent performance and robustness in control, and has been widely used in industrial process control.

The original GPC algorithm requires matrix inversion and large calculation, and it is not suitable for a real time system which needs quick response. What is worse, the original GPC may cause large amplitude overshoot sometimes and result in long time concussion.

In this paper, based on the original GPC, stair-like control scheme is used and it can avoid matrix inverse operations. Besides, the predicted future changes are considered as feedback. In this way, the overshoot can be suppressed. Better application of GPC in ATO system can be achieved.

\subsection{Basic principle of GPC}

The controlled object of GPC is described by CRIMA model:

$$
A\left(z^{-1}\right) y(k)=B\left(z^{-1}\right) u(k-1)+C\left(z^{-1}\right) \delta(k) / \Delta
$$

where, $A\left(z^{-1}\right), B\left(z^{-1}\right), C\left(z^{-1}\right)$ are polynomial of operator $z^{-1}, u(k)$ and $y(k)$ denote the input and output, $\Delta=1-\mathrm{z}^{-1}$ is the difference operator, $\delta(k)$ is the noise sequence.

In order to get the prediction of the forward $j$ step of the system, according to paper [6], the Diophantine equation is introduced.

$$
\begin{gathered}
1=E_{j}\left(z^{-1}\right) A\left(z^{-1}\right) \Delta+z^{-j} F_{j}\left(z^{-1}\right) \\
E_{j}\left(z^{-1}\right) B\left(z^{-1}\right)=G_{j}\left(z^{-j}\right)+z^{-j} H_{j}\left(z^{-1}\right)
\end{gathered}
$$

where, $j=1, \ldots, P, P$ is the prediction length, $E_{j}, F_{j}, G_{j}$ and $H_{j}$ are polynomials determined by the model parameters and the prediction length.

By means of the deformation and solution of the Diophantine equation, the predictive output formula is obtained as follows:

$$
y(k+j)=G_{j} \Delta u(k+j-1)+F_{j} y(k)+H_{j} \Delta u(k-1)+E_{j} \varepsilon(k+j)
$$

which can be expressed as vector form:

$$
\mathbf{Y}=\mathbf{G} \mathbf{U}+\mathbf{F} y(k)+\mathbf{H} \Delta u(k-1)+\mathbf{E}
$$

GPC uses minimum variance optimal control. Its objective function is:

$$
J=\mathrm{E}\left\{\left[\left(\mathbf{Y}-\mathbf{Y}_{r}\right)^{\mathrm{T}}\left(\mathbf{Y}-\mathbf{Y}_{r}\right)\right]+\lambda \mathbf{U}^{\mathrm{T}} \mathbf{U}\right\}=\mathrm{E}\left\{\sum_{\mathrm{j}=N_{1}}^{P}\left[y(k+j)-y_{r}(k+j)\right]^{2}+\sum_{\mathrm{j}=N_{1}}^{M} \lambda(j)[\Delta u(k+j-1)]^{2}\right\}
$$

where, $P$ is the prediction length, $M$ is the control length, and $y_{r}$ is the target value.

The optimal control rate can be obtained as follows:

$$
\mathbf{U}=\left(\lambda \mathbf{I}+\mathbf{G}^{\mathrm{T}} \mathbf{G}\right)^{-1} \mathbf{G}^{\mathrm{T}}\left[\mathbf{Y}_{r}-\mathbf{F} y(k)-\mathbf{H} \Delta u(k-1)\right]
$$

The definition of the first row of $\left(\lambda \mathbf{I}+\mathbf{G}^{\mathrm{T}} \mathbf{G}\right)^{-1} \mathbf{G}^{\mathrm{T}}$ is 


$$
p^{\mathrm{T}}=\left[p_{1}, p_{2}, \ldots, p_{P}\right]
$$

Then, the real time optimal control is

$$
\begin{gathered}
u(k)=u(k-1)+\Delta u(k) \\
\Delta u(k)=p^{\mathrm{T}}\left[\mathbf{Y}_{r}-\mathbf{F} y(k)-\mathbf{H} \Delta u(k-1)\right]
\end{gathered}
$$

\subsection{Stair-like control}

In the original GPC algorithm, there are a large number of matrix inverse operations, which will bring some security risks to the system. Therefore, based on the GPC, this paper uses the stair-like control scheme to convert the matrix inverse operation to the scalar inverse operation.

The step factor is $\beta \in(0,1)$, and the step control matrix is defined as follows:

$$
\mathbf{Q}^{\mathrm{T}}=\left[1,1+\beta, \ldots, 1+\sum_{i=1}^{M-1} \beta^{i}\right]
$$

We can get

$$
\begin{gathered}
\Delta u(k+j)=1+\sum_{i=1}^{j} \beta^{i} \Delta u(k) \\
\mathbf{U}^{\mathrm{T}}=\mathbf{Q}^{\mathrm{T}} \Delta u(k)=\left[1,1+\beta, \ldots, 1+\sum_{i=1}^{M-1} \beta^{i}\right] \times \Delta u(k)
\end{gathered}
$$

Let $\partial \mathbf{J} / \partial \mathbf{U}=0, \mathbf{G Q}=\mathbf{L}$, then,$$
\Delta u(k)=\left(\lambda \mathbf{Q}^{\mathrm{T}} \mathbf{Q}+\mathbf{L}^{\mathrm{T}} \mathbf{L}\right)^{-1} \mathbf{L}^{\mathrm{T}}\left[\mathbf{Y}_{r}-\mathbf{F} y(k)-\mathbf{H} \Delta u(k-1)\right]
$$$$
u(k)=u(k-1)+\Delta u(k)
$$

Due to the introduction of stair-like control, the input is planned in a ladder form and it makes the input changes in one direction over a period of time. In this way, it can greatly reduce the cost in changing and save energy. At the same time, the matrix inversion is converted to scalar operation and the actual calculation is greatly reduced. Therefore, the system has a faster response speed.

\subsection{Input increment feedback}

Generally, a first-order filter equation is set up according to the target value $y_{r}$. And the controlled variable can be set along the reference trajectory. But it is difficult to find the balance point between the tracking speed and the suppression of overshoot. If the controlled input at moment $k+1$ can be predicted at moment $\mathrm{k}$, then we can use the estimated value to make compensation to the input at moment $\mathrm{k}$. And the overshoot can be further suppressed.

Put the input increment $\Delta u(k)$ at moment $k$ into (13) and the next best predicted value $\hat{y}(k+1 \mid k)$ can be gained. According to original GPC, the following equation can be gained.

$$
\Delta u(k+1 \mid k)=p^{\mathrm{T}}\left[\mathbf{Y}_{r}-\mathbf{F} \hat{y}(k+1 \mid k)-\mathbf{H} \Delta u(k)\right]
$$

The feedback control input can be obtained by a simple weighted average

$$
u^{\prime}(k)=u(k)+\Delta u(k+1 \mid k) / 2
$$

By using the change trend of the optimal control value at moment $k+1$, the control input at moment $k$ is compensated to a certain degree. And it can restrain the overshoot of the output result.

\section{Simulation Study}

Based on the train predict model and the improved control algorithm in this paper, the simulation study is carried out. According to the running data collected from Hangzhou Line 4, the train model is established. The improved GPC algorithm is applied to control the train operation process. The effectiveness of the improved GPC algorithm is proved by the simulation results. 
Set the simulation parameters $P=20, M=10$, and the improved GPC algorithm is applied for the simulation. The tracking speed result is shown in Fig. 1.

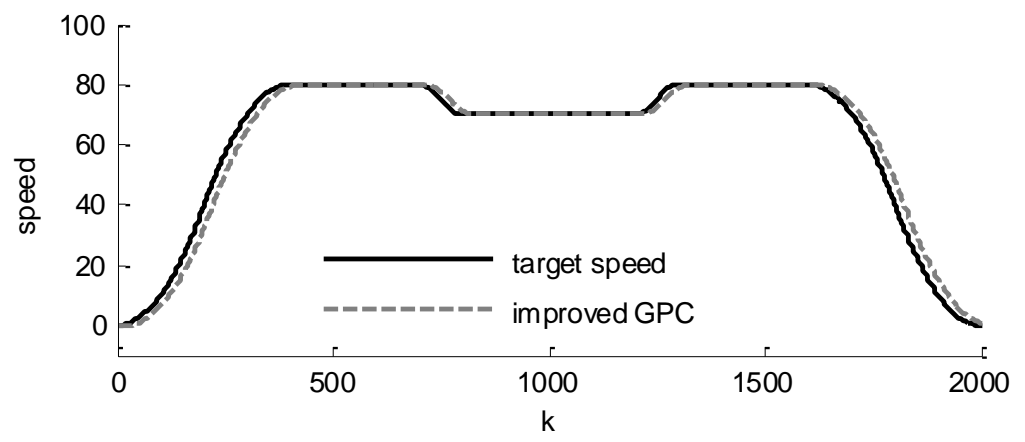

Fig. 1: Control result under the improved GPC

As is shown in Fig. 1, the controlled speed is very close to the target speed. The improved GPC algorithm can track the target speed smoothly and accurately.

Comparing the speed tracking results of the improved GPC and the original GPC, the compared results are shown as follows.

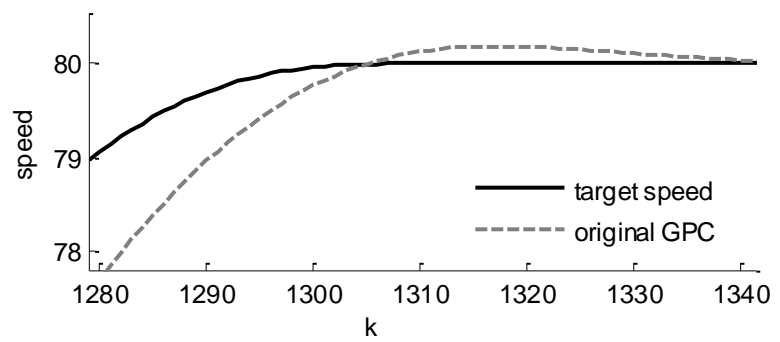

(a) overshoot of original GPC

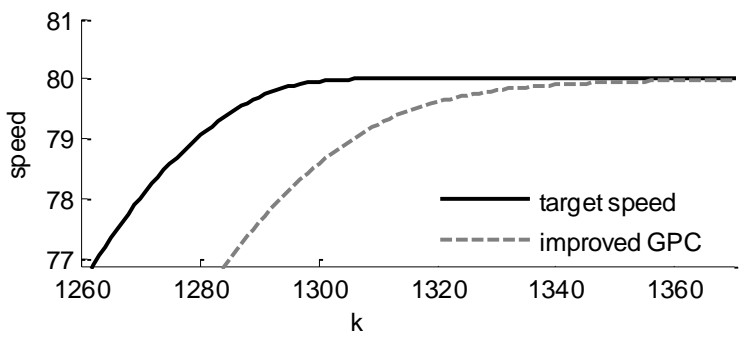

(b) overshoot of improved GPC

Fig. 1: Overshoot compared results

If there is a phenomenon of overshoot during the train running process, it may lead to over speed and cause emergency stop. It will affect the safety of the train system. As is shown in Fig 2, the controlled speed of the original GPC exceeds the target speed in a certain range. Meanwhile, the controlled speed of the improved GPC tracks the target speed stably and there is no overshoot. The improved GPC proposed in this paper has the advantage of suppressing overshoot.

The following figure shows the control results of the two methods under the changing operation conditions.
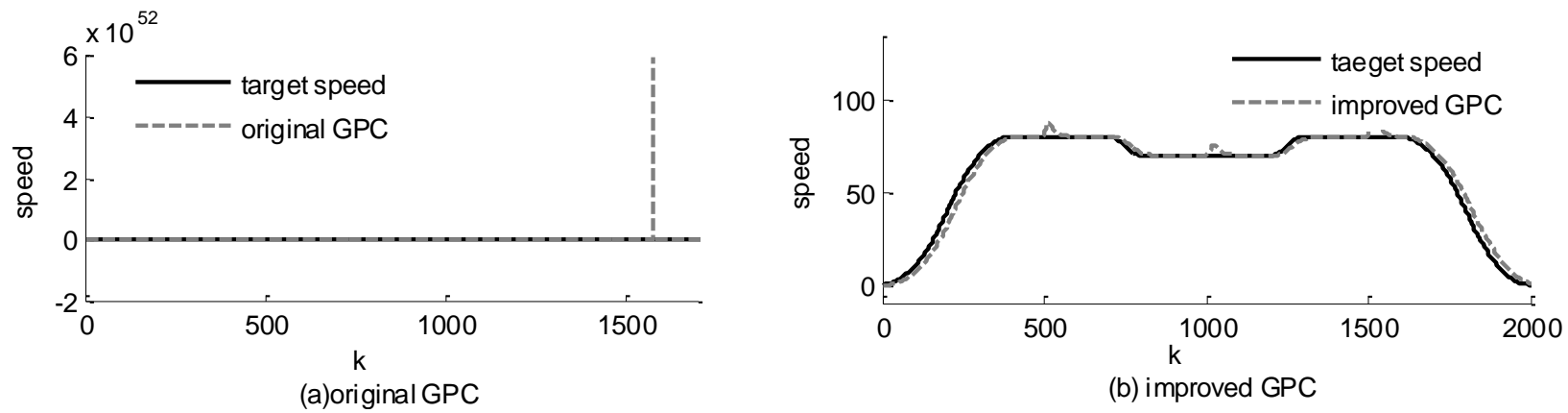

Fig. 2: Time-varying system control results

When the train runs in a time-varying condition, the original GPC will not work well because it requires matrix inversion and large calculation. Meanwhile, in the improved GPC algorithm, the stair-like control scheme is used and it can avoid solving the inverse of matrix. The target speed can be tracked in a timely manner, and the input can be obtained more accurately.

\section{Conclusion}


The basic GPC algorithm requires matrix inversion and large calculation, and it is not suitable for a real time system which needs quick response. In this paper, based on the original GPC, stair-like control scheme is adopted. Besides, the predicted future changes are considered as feedback. In this way, the computational cost of the algorithm is greatly reduced and the overshoot is suppressed. The simulation results show that the improved GPC controller as a good tracking ability for the target speed and it can meet the needs of ATO better. The improved GPC proposed in this paper has the advantage of suppressing overshoot and small error. Moreover, it can adapt to the changing operation conditions and meet the requirements of safety and comfort.

\section{Acknowledgements}

The authors wish to acknowledge Jing Zheng of Zhejiang University, who provided plenty of suggestions for this paper. This work is supported by the funding from the National Science and Technology Infrastructure Program of China under Grant 2015BAG19B03.

\section{References}

[1] L. J. Pei, "Research on Parameter Identification for Train Control Model and Its Online Learning Algorithms", M.S. Thesis, Beijing Jiaotong University, 2014.

[2] T. Tao and L.J. Huang, "A Survey of Control Algorithm for Automatic Train Operation", Journal of the China Railway Society, 2003, vol.25, pp.98-102.

[3] G. M. Gui and S. Z. Pu, "Simulation of ATO System Based on Fuzzy Generalized Predictive Control Algorithm", Urban Mass Transit, 2014, vol. 17, pp. 69-73.

[4] Y. Li and J. M. Yang, "Study on Controlling Increment in Generalized Predictive Control", Mechanical Engineering \& Automation, 2007, vol. 5, pp. 45-46.

[5] Y. H. Hu and X. L. Jia, "Summarization of Generalized Predictive Control", Information and Control, 2000, vol.29, pp.248-255.

[6] Z. W. Chen, "Research on Direct Generalized Predictive Control”, Ph.D. Thesis, Yanshan University, 2007. 